\title{
Closed model for granular compaction under weak tapping
}

\author{
J. Javier Brey* and A. Prados ${ }^{\dagger}$ \\ Física Teórica, Universidad de Sevilla, Apartado de Correos 1065, E-41080 Sevilla, Spain
}

(Received 25 June 2003; published 20 November 2003)

\begin{abstract}
A one-dimensional lattice model is formulated to study tapping dynamics and the long time steady distribution in granular media. The dynamics conserves the number of particles in the system, and density changes are associated with the creation and destruction of empty sites. The model is shown to be consistent with Edwards' thermodynamics theory of powders. The relationship with lattice models in which the number of particles is not conserved is discussed.
\end{abstract}

DOI: 10.1103/PhysRevE.68.051302

PACS number(s): 45.70.Cc, 05.50.+q, 81.05.Rm

\section{INTRODUCTION}

In the last few years, a great deal of effort is being made trying to understand the physical mechanisms leading to compaction in weakly vibrated granular systems, and the properties of the steady state eventually reached in the long time limit. This has been prompted and stimulated by the seminal papers of the Chicago group reporting experimental results of compaction [1-3]. Granular compaction consists of the increase in the density, starting from an initial lowdensity state, as a consequence of external excitations, usually vertical shakes or taps. Every tap is followed by a free relaxation, so that in the process the system goes through a series of blocked configurations.

Starting from an "ergodic hypothesis" for powders, based on the extensive, global, character of the dynamics induced by shaking, Edwards and co-workers [4] have formulated a microscopic theory for the steady state of vibrated granular media that is similar to conventional statistical mechanics. Moreover, they assume that the steady state is fully characterized by the volume of the system, which then plays a role analogous to that of the energy in the usual thermal systems. This provides the "microcanonical" description. The associated "canonical" probability distribution is obtained by maximizing the statistical entropy under the condition that the average volume is given. Of course, the probability of a given configuration depends only on its volume. The parameter conjugated of the volume, similar to the thermal temperature, was named compactivity by the authors in Ref. [4].

Up to now, there has been no definite experimental test of the above thermodynamic theory of powders. The measure of the compactivity, or the entropy, of a granular system seems a rather difficult task not only in real experiments but also in realistic models, although some procedures have been proposed. They are based on the determination of the average volume and its fluctuations as a function of the control parameter of the shaking, e.g., the vibration intensity [1,5]. From these two functions, the compactivity can be obtained, in principle, by integration, although this program is hard to carry out in practice due to the uncertainty of the measurements. Another alternative way, this one based on the gener-

\footnotetext{
*Electronic address: brey@us.es

†Electronic address: prados@us.es
}

alization of the Einstein relation between diffusivity and mobility, has been recently discussed and analyzed in a system of inelastic hard spheres by means of molecular dynamics simulations [6].

On the other hand, the validity of Edwards' theory has been studied in the context of several simple models, with different underlying physical mechanisms. It has been found that the results for Tetris and spin-glass models [7-9] are consistent with the theory. In these systems, the number of particles is fixed but most of the results are numerical, due to the complexity of the models used. One-dimensional Ising models, with or without kinetic constrains, have also been considered [10-14], because they are simple enough as to allow a detailed analytical study in many cases. For weak tapping, agreement with Edwards' theory was found again, although discrepancies show up in the limit of strong tapping. Quite interestingly, all the Ising-like models in Refs. [10-14] have been formulated as open systems. The number of particles does not remain constant, but it changes along the compaction process, as a consequence of adsorptiondesorption events from a theoretical particle reservoir in contact with the system. Instead, it is the volume that is kept fixed, in this way leading to the variation of the density. Then, although it is true that the steady distribution of these models can be considered as a "grand canonical" ensemble generalization of the theory, it is also clear that it is not characterized by the compactivity (temperature) but by another parameter playing the role of the chemical potential. This difference is evidently relevant when trying to relate any of them with the characteristics of the vibration process, e.g., its intensity. Beyond that, the distinction might become conceptually crucial when dealing with granular mixtures and segregation phenomena. In that case, each of the different species is going to have its own analogous of the chemical potential parameter [15]. Whether or not it is also needed to consider different compactivities (temperatures), as it has been suggested recently [16], is a different question.

The aim of this paper is to present a closed, constant number of particles, one-dimensional lattice model for compaction. Again the model is simple enough as to be analytically tractable. During the tapping process, particles diffuse and also empty sites (holes) are created and destroyed in the system, according to well defined rules. The latter are chosen to mimic, in a crude way, what happens in real compaction experiments. More precisely, the model tries to represent a 
vertical section of a vibrated two-dimensional system. In a shake, the length of the section can increase because empty regions (holes) are created between the particles. These regions can be used for the particles to diffuse. Afterwards, once the shake has ended, the system tries to compact due to the action of gravity. This is accomplished in the model by means of the elimination of holes. But, because of the geometrical constrains following from excluded volume effects of the hard particles in the neighboring vertical sections, not all the holes can be destroyed in the free evolution. Only large enough empty regions can be reduced. As a consequence of the combination of a tap and the next free relaxation, the length can increase in some regions of the system and decrease in others. The net balance determines the global behavior of the system in the compaction process.

The plan of the paper is as follows. In Sec. II, the model is formulated at the mesoscopic level of description by means of a master equation for the transition probability. This equation is exactly solved for the steady distribution in Sec. III, and the associated macroscopic description is discussed in Sec. IV, where it is shown to be in agreement with Edwards' thermodynamic description. The compactivity is identified in terms of the parameters characterizing the mesoscopic dynamics of the model. Also, the distribution of domains is derived there. Section V contains a detailed discussion of the relationship between closed and open models, and between the compactivity and fugacity parameters. The paper ends with a short summary and some additional discussions.

\section{THE MODEL}

We consider a one-dimensional lattice having $N+1$ particles. The number of sites in the lattice is variable and changes with time accordingly with the rules to be specified below. Those sites that are not occupied by a particle are said to be empty or, equivalently, being a hole.

The dynamics of the system is defined trying to mimic tapping experiments for the study of compaction in granular media [1-3]. These experiments typically involve two different series of processes of quite different nature. The system is submitted to taps or pulses separated by time intervals for which the system is allowed to relax freely, until being trapped in a metastable configuration. Therefore, each tap starts in the metastable configuration reached in the previous free relaxation. The taps are characterized by their duration and their amplitude.

Physically, the effect of the taps is to decrease the local density in some regions of the system, moving grains from their metastable positions, and allowing a posterior reordering in the free relaxation. We will specify first the dynamics during the relaxation processes, since it leads to identifying the possible metastable, or blocked, configurations of the model. It will be assumed that in the free relaxation, the system tries to reduce its length by eliminating some of the empty sites of the lattice. More precisely, whenever there is a group of nearest neighbor holes, all except one are eliminated. These are the only processes taking place in the free relaxation, and have probability one. Therefore, the number (a)

$$
\begin{aligned}
& \mathrm{OOXO} \stackrel{\alpha}{\longrightarrow} \mathrm{OXXO} \stackrel{1 / 2}{\longrightarrow} \mathrm{OXOO} \\
& \mathrm{OxOO} \stackrel{\alpha}{\longrightarrow} \mathrm{OXXO} \stackrel{1 / 2}{\longrightarrow} \mathrm{OOXO}
\end{aligned}
$$

(b)

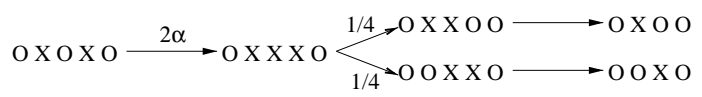

(c)

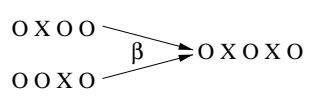

FIG. 1. Elementary rearrangements of the system in a single tap and the following free relaxation in the weak vibration limit. The trajectories leading to a final state identical to the initial one are not shown.

of particles is conserved in the relaxation, but the length of the system, measured by the total number of sites, is in general reduced.

As a consequence of the above dynamics for relaxation, the metastable configurations of the model are characterized by having all the holes surrounded by two particles, i.e., the holes are isolated. In order to displace the system from one of these states, it has to be externally perturbed, for instance by means of a tap. To complete the description of the dynamics of our model in a compaction experiment, the possible transitions taking place during a tap and starting at a metastable configuration, have to be identified and their probabilities specified. Two kinds of elementary processes will be considered. Each of them will be discussed separately in the following.

First, a particle can be transiently desorbed from the lattice and posteriorly adsorbed in an empty site in the neighborhood of its previous position. This process is restricted by the following rule. A particle can be desorbed from a site during the tap only if at least one of its nearest neighbor sites is empty. More precisely, the probability for these events is proportional to the number of nearest neighbor holes of the particle being desorbed. This restriction tries to naively model the short range constrains making difficult structural rearrangements in granular materials. Then, during a tap, the probability of desorption of a particle having only one nearest neighbor hole is $\alpha$, while it is $2 \alpha$ if it is surrounded by two holes. Afterwards, the particle is reabsorbed either in its own original site or in any of the nearest neighbor holes, with a probability that is proportional to the number of holes next to the site considered. In Fig. 1, cases $(a)$ and $(b)$ involve processes starting with the transitory desorption of a particle. Particles and holes are represented by circles and crosses, respectively. In the case referred to as $(b)$ in the figure, the elimination of a hole happening in the next free evolution has been also indicated. It is seen that the net result of the series of events taking place during the tap and the free relaxation is, in this case, the destruction of a lattice site, with the consequent decrease of the lattice length.

During the tap, the creation of an empty site or hole is also possible, but only between two nearest neighbor particles located at one of the ends of a domain of at least two particles. The probability of the corresponding elementary events, referred to as case $(c)$ in Fig. 1 , is $\beta$. Note that these processes of hole creation are just the inverse of those pro- 
TABLE I. Transition probabilities for the elementary rearrangements taking place in a single tap in the weak vibration regime.

\begin{tabular}{ccc}
\hline \hline Initial state & Final state & Probability \\
\hline OOXO & OXOO & $\alpha / 2$ \\
OXOO & OOXO & $\alpha / 2$ \\
OXOXO & OXOO & $\alpha / 2$ \\
OXOXO & OOXO & $\alpha / 2$ \\
OXOO & OXOXO & $\beta$ \\
OOXO & OXOXO & $\beta$ \\
\hline \hline
\end{tabular}

ducing the destruction of a hole.

Furthermore, it will be assumed that only one transition takes place at the most in every region on the system during each tap, i.e., no site is involved into two different processes in the same perturbation of the system. Physically, this hypothesis implies to consider the limit of weak and short taps $[10,14]$. In summary, the dynamics of the model in the shaking process is defined by the effective transitions given in Table I, describing the combined events associated with a tap and the next free relaxation. The transitions only affect the clusters shown, and their probabilities are independent of the configuration of the remainder of the system.

To formulate the model in a more mathematical language, and also to characterize the metastable configurations, it is convenient to define a set of variables $\mathbf{n} \equiv\left\{n_{1}, \ldots, n_{N}\right\}$. The variable $n_{i}$ takes the value unity if there is a hole next to the right of particle $i$, while it vanishes if there is no hole, i.e., if particles $i$ and $i+1$ are in nearest neighbor sites. By definition, it is assumed that there is no hole to the left of particle 1 nor to the right of particle $N+1$. Both particles define the boundaries of the system. It is easily realized that this property is preserved by the dynamics of the system under tapping, as defined above. Then, we have established a one to one relationship between a set of $N$ variables taking values 0 and 1 and the metastable configurations of the model.

The transition probabilities in Table I can be expressed in terms of the $n_{i}$ variables. Denoting $R_{i} \mathbf{n}$ $\equiv\left\{n_{1}, \ldots, R_{i} n_{i}, \ldots, n_{N}\right\}$, with $R_{i} n_{i}=1-n_{i}$, the probability $W\left(\mathbf{n}^{\prime} \mid \mathbf{n}\right)$ of the several events going from configuration $\mathbf{n}$ to configuration $\mathbf{n}^{\prime}$ in the effective dynamics describing shaking process are

$$
\begin{aligned}
& W\left(R_{i} R_{i+1} \mathbf{n} \mid \mathbf{n}\right)=\frac{\alpha}{2}\left[n_{i}\left(1-n_{i+1}\right)+\left(1-n_{i}\right) n_{i+1}\right], \\
& \begin{aligned}
W\left(R_{i} \mathbf{n} \mid \mathbf{n}\right)= & \frac{\alpha}{2}\left(n_{i-1} n_{i}+n_{i} n_{i+1}\right)+\beta\left[n_{i-1}\left(1-n_{i}\right)\right. \\
& \left.+\left(1-n_{i}\right) n_{i+1}\right] .
\end{aligned}
\end{aligned}
$$

Equation (1) corresponds to diffusive events, while the first and second terms on the right-hand side (rhs) of Eq. (2) correspond to the destruction and creation of holes, respectively. The Markov process defined by these transition probabilities is irreducible, i.e., all the metastable configurations of the lattice are connected by a chain of transitions with nonzero probability. To verify this property, we begin by

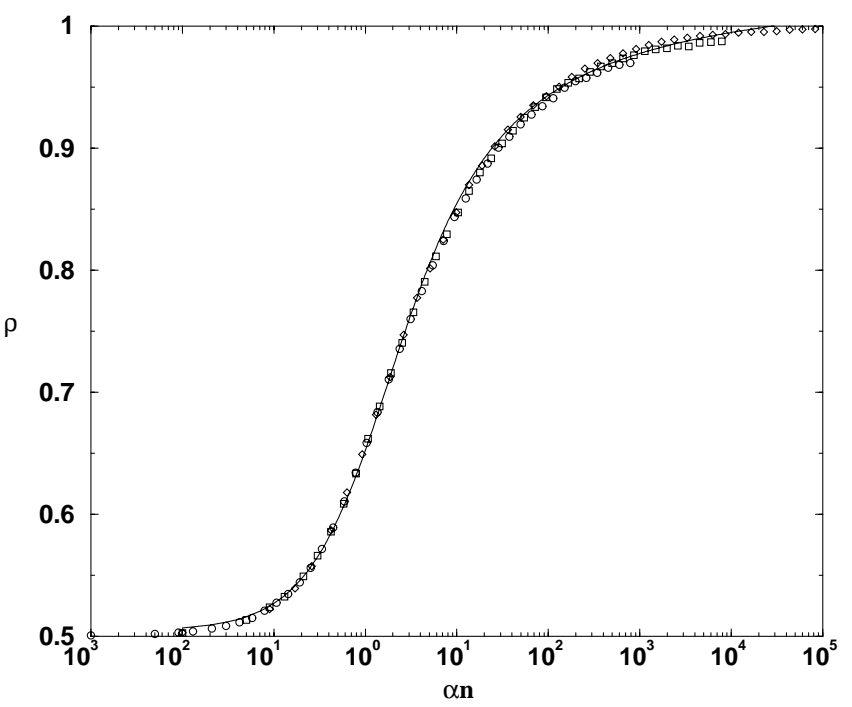

FIG. 2. Evolution of the density of particles, as a function of the scaled time defined in the text. The curves correspond to the pairs of values $\alpha=10^{-3}, \quad \beta=10^{-5}$ (circles), $\alpha=10^{-2}, \quad \beta=5 \times 10^{-5}$ (squares), and $\alpha=10^{-2}, \beta=5 \times 10^{-6}$ (diamonds), while the solid line is the best inverse logarithmic fit, Eq. (3), with the parameters given in the text.

noting that any metastable configuration can be connected with the configuration characterized by having just a hole located next to the right of a given fixed particle. This is because holes can be moved through particles by means of diffusive events, so that the two consecutive holes can always be located on both sides of the same particle. Afterwards, one of the holes can be eliminated by a type $(b)$ event of Fig. 1. This procedure can be repeated until there is only a hole in the lattice, which can then be diffused to the desired site. This proves the above statement. But, since each effective transition have its inverse also with nonzero probability, the above paths can also be reversed, concluding that all the metastable configurations are connected. The irreducibility property of the Markov process implies that there is a unique steady probability distribution for the process [17]. This distribution will be explicitly obtained in the following section.

In Fig. 2, the relaxation of the particle density is shown as a function of the "scaled time" $\tau=\alpha n$, where $n$ is the number of taps before measuring the density for different values of $\alpha$ and $\beta$. The initial state for all the curves was the least dense metastable configuration, $\rho=0.5$, in which there is a hole between every two particles. In all the reported cases $\beta \ll \alpha$, so that processes decreasing the density of particles are only relevant when the system is near the most compact state, $\rho=1$. Moreover, as $\beta \ll \alpha \ll 1$, there is a universal behavior up to a very large number of taps $t=\mathcal{O}\left(\alpha^{-1}\right)$, i.e., $\alpha n$ $=\mathcal{O}(1)$. For longer times, when processes decreasing the density become relevant, $n \geqslant \mathcal{O}\left(\beta^{-1}\right)$, the system approaches a steady state characterized by the ratio $\beta / \alpha$. The observed universal scaled curve is very well described by the four-parameter empirical law

$$
\rho(t)=\rho_{\infty}-\frac{\delta \rho_{\infty}}{1+B \ln \left(1+\frac{\tau}{\tau_{c}}\right)},
$$


with $\rho_{\infty}=1.04, \delta \rho_{\infty}=0.54, B=1.17$, and $\tau_{c}=2.63$. As it is the case with the experimental data [1] and also with numerical results from other simple models $[10,18]$, the logarithmic fit is not expected to give the correct asymptotic density of particles. In fact, in our case it is $\rho_{\infty}>1$, which is clearly unphysical. A similar behavior of $\rho_{\infty}$ was found in Ref. [10]. Also, values of $\rho_{\infty}$ larger than the random close packing limit have been reported from the fit of experimental data [1].

\section{THE STEADY DISTRIBUTION}

To find the steady distribution of the Markov process describing the effective dynamics of the model, we are going to assume it verifies detailed balance. Of course, this has to be justified a posteriori by showing that such a distribution exists. Therefore, we look for a time-independent distribution $p^{(s)}(\mathbf{n})$ having the property

$$
W\left(\mathbf{n}^{\prime} \mid \mathbf{n}\right) p^{(s)}(\mathbf{n})=W\left(\mathbf{n} \mid \mathbf{n}^{\prime}\right) p^{(s)}\left(\mathbf{n}^{\prime}\right)
$$

for all configurations $\mathbf{n}$ and $\mathbf{n}^{\prime}$. A direct first consequence of this equation is that all the metastable configurations with the same number of holes have the same probability in the steady state. This follows from the fact that they are connected by diffusive events and diffusion is isotropic in the effective dynamics, as seen in Table I. Therefore, the distribution function verifying Eq. (4) can only depend on the number of holes

$$
N_{H}=\sum_{i}^{N} n_{i},
$$

in the configuration, but not on their spatial distribution. So, we can write

$$
p_{N_{H}}^{(s)}(\mathbf{n})=\frac{f\left(N_{H}\right)}{Z},
$$

where the number of holes, $N_{H}$, in the configuration $\mathbf{n}$ has been made explicit in the notation, $f\left(N_{H}\right)$ is a function to be determined, and $Z$ denotes a normalization constant,

$$
Z=\sum_{\mathbf{n}} f\left(N_{H}\right)
$$

Still remains to be analyzed, if Eq. (6) can be made compatible with Eq. (4), when particularized for effective events increasing (and decreasing) the number of holes. The latter reads

$$
\beta p_{N_{H}}^{(s)}(\mathbf{n})=\frac{\alpha}{2} p_{N_{H}+1}^{(s)}\left(\mathbf{n}^{\prime}\right) .
$$

Here $\mathbf{n}^{\prime}$ is a configuration differing from $\mathbf{n}$ by the creation of a hole. Use of Eq. (6) gives

$$
\frac{f\left(N_{H}+1\right)}{f\left(N_{H}\right)}=\frac{2 \beta}{\alpha}
$$

and, by iteration,

$$
f\left(N_{H}\right)=C\left(\frac{2 \beta}{\alpha}\right)^{N_{H}}
$$

for $N_{H} \geqslant 1, C$ being an arbitrary constant that will be taken equal to unity. In this way, we have proven that the system has the property of detailed balance and that its steady distribution is given by

$$
\begin{gathered}
p_{N_{H}}^{(s)}(\mathbf{n})=\frac{\gamma^{-N_{H}}}{Z}, \\
Z=\sum_{\mathbf{n}} \gamma^{-N_{H}}=\sum_{N_{H}=1}^{N} \Omega_{N}\left(N_{H}\right) \gamma^{-N_{H}},
\end{gathered}
$$

where $\gamma=\alpha / 2 \beta$ and $\Omega_{N}\left(N_{H}\right)$ is the number of metastable configurations of the lattice having $N_{H}$ holes and, of course, $N+1$ particles. It is worth remarking that no approximation has been done in order to derive the steady distribution, Eq. (11), i.e, it is valid for any value of $\gamma$. The steady average number of holes and its dispersion can be evaluated from $Z$ by

$$
\begin{gathered}
\left\langle N_{H}\right\rangle_{s} \equiv \sum_{\mathbf{n}} N_{H} p_{N_{H}}^{(s)}(\mathbf{n})=-\frac{\partial \ln Z}{\partial \ln \gamma} \\
\left\langle\left(\Delta N_{H}\right)^{2}\right\rangle_{s} \equiv\left\langle N_{H}^{2}\right\rangle_{s}-\left\langle N_{H}\right\rangle_{s}^{2}=\frac{\partial^{2} \ln Z}{\partial(\ln \gamma)^{2}}=-\frac{\partial\left\langle N_{H}\right\rangle_{s}}{\partial \ln \gamma} .
\end{gathered}
$$

A simple combinatorial argument gives

$$
\Omega_{N}\left(N_{H}\right)=\frac{N !}{N_{H} !\left(N-N_{H}\right) !}
$$

and substitution of this expression into Eq. (12) yields

$$
Z=\left(1+\frac{1}{\gamma}\right)^{N}-1
$$

Therefore, it follows by application of Eq. (13) that, in the limit of large $N$,

$$
\left\langle N_{H}\right\rangle_{s}=\frac{N}{1+\gamma} .
$$

The right-hand side of Eq. (17) is a monotonic decreasing function of $\gamma$ for fixed number of particles $N$, i.e., the length of the system decreases as $\gamma$ increases. Therefore, $\gamma^{-1}$ plays a role similar to the vibration intensity in real granular experiments of compaction in the model. It follows that, in the physical image depicted by the present model, the probability of diffusion process $\alpha$ is expected to grow faster with the vibration intensity than the probability of creation of holes $\beta$, at least in the weak tapping limit.

The length (volume) of a configuration is given by

$$
L=N_{H}+N,
$$

and the average particle density is 


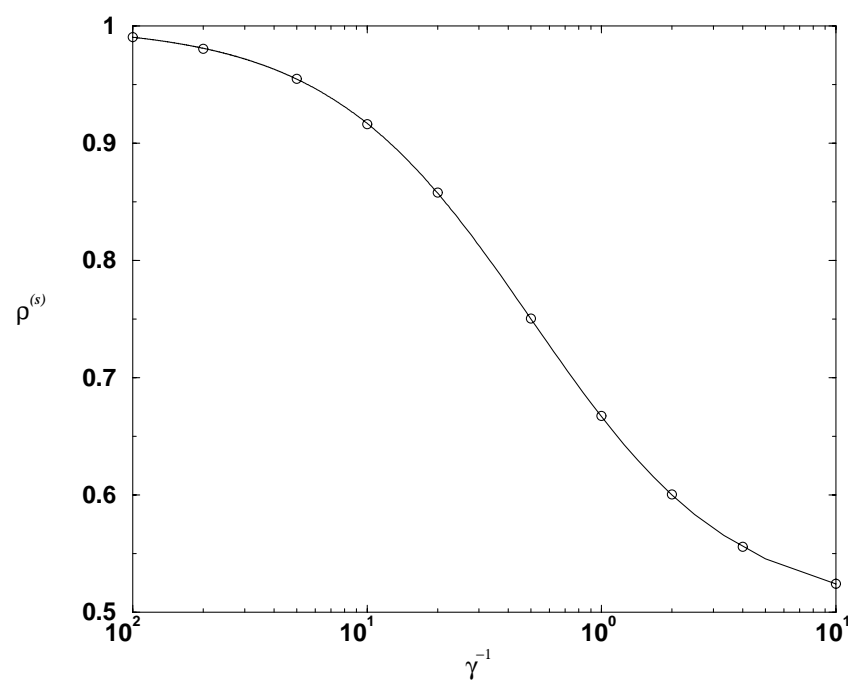

FIG. 3. Comparison between the numerical values of the steady density of particles and the theoretical prediction given by Eq. (19).

$$
\rho^{(s)}=\frac{N}{\langle L\rangle_{s}}=\frac{N}{\left\langle N_{H}\right\rangle_{s}+N}=\frac{1+\gamma}{2+\gamma} .
$$

In Fig. 3, the numerical values for the steady density of particles, obtained by Monte Carlo simulation of the model, are compared with the theoretical prediction, Eq. (19), and an excellent agreement is found. The specific length per particle, in site units, is the inverse of the particle density,

$$
\langle l\rangle_{s} \equiv \frac{N+\left\langle N_{H}\right\rangle_{s}}{N}=\frac{2+\gamma}{1+\gamma} .
$$

Its dispersion is obtained from Eqs. (14) and (16),

$$
N\left\langle(\Delta l)^{2}\right\rangle_{s}=\left(2-\langle l\rangle_{s}\right)\left(\langle l\rangle_{s}-1\right),
$$

presenting a maximum for $\langle l\rangle_{s}=3 / 2$, i.e., when the average number of holes is $N / 2$ and the density of particles $\rho^{(s)}$ $=2 / 3$, i.e., $\gamma=1$. The numerical evaluation of the length fluctuations is compared with the theoretical prediction, as given by Eq. (21), in Fig. 4. Again, a very good agreement is observed for the range of "vibration intensities" $\gamma^{-1}$ plotted. Outside this window of vibration intensities, the length fluctuations are very small and, therefore, rather hard to measure in the simulations.

\section{THERMODYNAMIC DESCRIPTION}

Following Edwards and co-workers ideas [4], the steady distribution (11) can be expressed in the canonical form

$$
p_{N_{H}}^{(s)}(\mathbf{n})=\frac{e^{-N_{H} / X}}{Z}, \quad Z=\sum_{N_{H}} \Omega_{N}\left(N_{H}\right) e^{-N_{H} / X},
$$

where

$$
X=(\ln \gamma)^{-1}
$$

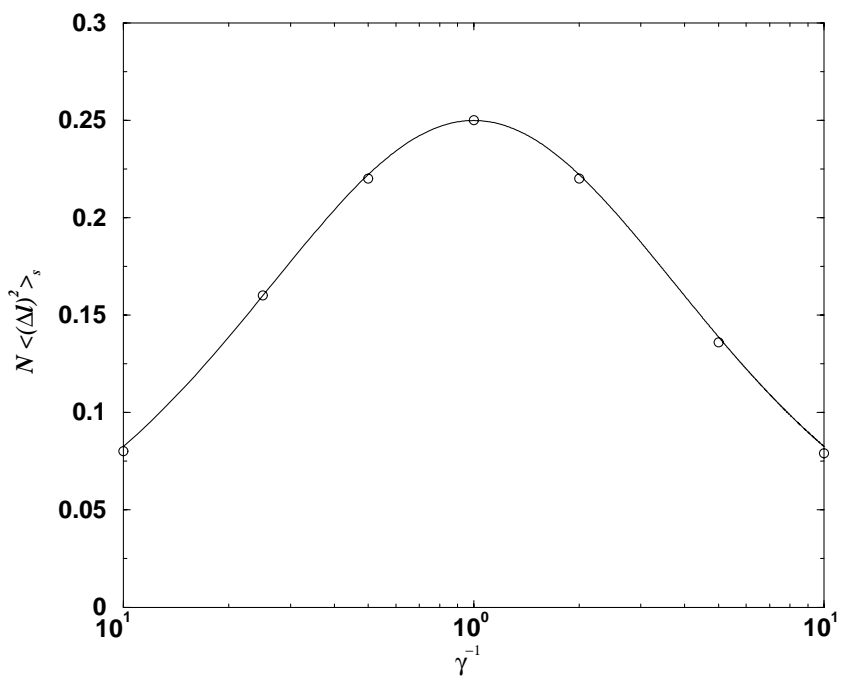

FIG. 4. Comparison between the numerical evaluation of the length fluctuations and the theoretical prediction given by Eq. (21).

is the so-called compactivity. It is the conjugated thermodynamic parameter of the volume in gently vibrated granular systems, in an analogous way as the temperature is the conjugate of the energy in usual thermal systems. Note that, in Eq. (22), the ratio $N_{H} / X$ can be replaced in both the numerator and the denominator by $L / X$, where $L$ is the length (volume) of the configuration, as defined in Eq. (18). The structure of the above steady distribution is consistent with the two main ingredients of Edwards' theory, namely, that the measure over metastable configurations is flat, and that there is a unique parameter the volume, characterizing the macroscopic state of the system. Let us point out that, very recently, the theory has been extended to include several macroscopic control parameters, in an effort to explain the discrepancies observed in some models with strong tapping $[12,19]$, and also segregation patterns in binary models [16]. It is clear that such an extension does not apply to our model, which is designed to describe compaction in one-component systems under weak tapping. In the same context, the expression of the compactivity in Eq. (23) deserves some comments. Although $X$ can be formally negative, for values $\gamma<1$, it is quite doubtful that this fact be physically relevant, since this range of values of $\gamma$ corresponds to strong tapping, leading to low stationary densities, namely, with an average number of holes $\left\langle N_{H}\right\rangle_{s}>N / 2$. The possibility of negative values of the compactivity has been also found in other simple models $[10,20]$, and it is associated with the existence of a maximum length for the metastable configurations.

In the limit $\gamma \rightarrow \infty$, i.e., asymptotically weak tapping, the steady concentration of particles, $\rho^{(s)}$, given by Eq. (19) can be approximated by

$$
\rho^{(s)} \simeq 1-\frac{1}{\gamma}
$$

and, using the definition in Eq. (23),

$$
X^{-1}=-\ln \left[1-\rho^{(s)}\right]
$$


This relation between the compactivity and the steady density has also been found in a model with facilitated dynamics having a variable number of particles and fixed volume [10], and a similar behavior has been reported from the analysis of experimental data [3].

An "entropy" $S$ associated with the distribution $p^{(s)}$ can be defined in the usual way,

$$
S=-\sum_{\mathbf{n}} p^{(s)}(\mathbf{n}) \ln p^{(s)}(\mathbf{n}),
$$

and use of Eq. (22) gives

$$
S=\frac{\left\langle N_{H}\right\rangle_{s}}{X}+\ln Z
$$

Taking into account Eq. (13), it is easily verified that

$$
\frac{\partial S}{\partial\langle L\rangle_{s}}=\frac{\partial S}{\partial\left\langle N_{H}\right\rangle_{s}}=\frac{1}{X}
$$

consistently with the physical meaning of the compactivity as discussed above. Given that the macroscopic state of the system is characterized by a single parameter, it is possible to express the entropy in terms of only the density of particles, or the intensity parameter $\gamma$, or the compactivity. Then, for instance, in the limit of large $N$ the entropy can be written as

$$
\frac{S}{N}=\frac{1}{X\left(1+e^{1 / X}\right)}+\ln \frac{1+e^{1 / X}}{e^{1 / X}} .
$$

In addition to the global properties considered up to now, it is also possible to obtain information about the domain structure of the steady configurations. In particular, we are going to derive here the probability distribution for the number of particles in a domain. A domain of size $r$ is defined as a cluster of $r$ particles, i.e., two holes with $r$ particles in between. First, we consider the probability $F_{r}^{(s)}$ of finding a local domain of size $r$,

$$
F_{r}^{(s)} \equiv\left\langle n_{k}\left(1-n_{k+1}\right) \cdots\left(1-n_{k+r-1}\right) n_{k+r}\right\rangle_{s},
$$

with $r \leqslant 1$. Use of Eqs. (11) and (15) yields

$$
F_{r}^{(s)}=\frac{1}{Z} \sum_{N_{H}=0}^{N-r-1} \Omega_{N-r}\left(N_{H}\right) \gamma^{-2-N_{H}}=\gamma^{-2}\left(\frac{\gamma}{1+\gamma}\right)^{r+1},
$$

where the limit of large $N$ has been considered once again. Then, the probability of a domain of size $r, P(r)$, is given by the conditional probability of finding a cluster of $r$ consecutive particles plus a hole to the right of a given hole, i.e.,

$$
P(r)=\frac{F_{r}^{(s)}}{\left\langle n_{k}\right\rangle_{s}}=\frac{N}{\left\langle N_{H}\right\rangle_{s}} F_{r}^{(s)}=\frac{\gamma^{r-1}}{(1+\gamma)^{r}} .
$$

This distribution is correctly normalized:

$$
\sum_{r=1}^{\infty} P(r)=1
$$

It is instructive to express the distribution of domain sizes in terms of the average length per particle, $\langle l\rangle_{s}$. This is easily accomplished by means of Eq. (20), obtaining

$$
P(r)=\left(2-\langle l\rangle_{s}\right)^{r-1}\left(\langle l\rangle_{s}-1\right) .
$$

\section{RELATIONSHIP BETWEEN CLOSED AND OPEN MODELS FOR COMPACTION}

In the preceeding section, we have introduced the compactivity $X$ from the canonical form of the steady probability distribution, Eq. (22). In the Edwards and co-workers formulation of the granular thermodynamic theory [4], the compactivity was defined by

$$
X^{-1}=\left(\frac{\partial S}{\partial V}\right)_{N},
$$

where the entropy $S$ is given by

$$
S=\ln \Omega_{N},
$$

$\Omega_{N}$ being the number of blocked configurations or, in the language used in this paper, metastable states. In Eq. (35), the number of particles in the system is kept constant. The quantity $\Omega_{N}$ for the model considered in this paper is given by Eq. (15) and for $N \gg 1, N_{H} \gg 1$, Eq. (35) leads to

$$
X^{-1}=\ln \frac{N-N_{H}}{N_{H}} .
$$

This is the microcanonical (constant volume) version of the canonical (constant compactivity) expressions (17) and (23). In fact, combination of these two latter expressions gives

$$
X^{-1}=\ln \frac{N-\left\langle N_{H}\right\rangle_{s}}{\left\langle N_{H}\right\rangle_{s}} .
$$

The expression equivalent to Eq. (36) in the canonical ensemble is Eq. (26). It is evident that, in the limit of large systems, it is consistent with the definition of $X$ in Eq. (35).

In several proposed models for compaction, lattices with a fixed number of sites, i.e., fixed length, have been considered. The dynamics is defined involving elementary processes associated with the adsorption and desorption of particles in such a way that the number of particles in the lattice changes along the shaking experiment. This is the mechanism for which the density in the system varies with time. In particular, several models leading to similar kind of metastable configurations as in the model in this paper have been discussed in detail $[10-12,19,14]$. Then, aside from details that are irrelevant for the following analysis, the number of blocked configurations is given by Eq. (15), which we rewrite in the form

$$
\Omega_{L}\left(N_{H}\right)=\frac{\left(L-N_{H}\right) !}{N_{H} !\left(L-2 N_{H}\right) !},
$$


where the number of sites of the lattice $L$ is now considered as fixed and $L / 2 \geqslant N_{H} \geqslant 1$. Moreover the steady distribution, in the weak tapping limit was found to have the form

$$
p^{(s)^{\prime}}(\mathbf{n})=\frac{\eta^{-N_{H}}}{Z^{\prime}}
$$

with

$$
Z^{\prime}=\sum_{N_{H}=1}^{L / 2} \Omega_{N}\left(N_{H}\right) \eta^{-N_{H}},
$$

and $\eta$ is a given parameter, depending on the specific model, and characterizing the dynamical events in the system under shake. Then, from Eq. (40) a compactivity $X^{\prime}$ was identified as

$$
X^{\prime}=(\ln \eta)^{-1}
$$

This definition is equivalent to

$$
X^{\prime-1}=\left(\frac{\ln \Omega_{L}\left(N_{H}\right)}{\partial N_{H}}\right)_{L}=-\left(\frac{\partial S}{\partial N}\right)_{L},
$$

or, using Eq. (39),

$$
X^{\prime-1}=\ln \frac{\left(N-N_{H}\right)^{2}}{N_{H} N} .
$$

This expression differs from Eq. (37) except in the limit of high density $N_{H} / N \ll 1$, in which both reduce to $\ln \left(N / N_{H}\right)$, but this only indicates that the same density is obtained in this limit if $X=X^{\prime}$. Nevertheless, it must be stressed that $X$ is associated to tapping processes at constant number of particles, while $X^{\prime}$ describes processes at constant volume. Equivalently, $X$ characterizes ensembles with fixed $N$, and $X^{\prime}$ ensembles with fixed $L$. In this context, their physical nature is rather different. The parameter $X$ is the compactivity introduced by Edwards and, on the other hand, $\eta^{-1}$, related with $X^{\prime}$ by Eq. (42), plays the role of a fugacity for the particles. In terms of the entropy, $X$ and $X^{\prime}$ are related by

$$
X^{-1}=X^{\prime-1}+\left(\frac{\partial S}{\partial N}\right)_{N_{H}} .
$$

\section{CONCLUSION}

In this paper, a one-dimensional model for compaction in granular media has been presented. One of its main features, as compared with previous Ising-like models, is that the time evolution under tapping conserves the number of particles in the system, while it is the volume that changes in the compaction process. This is in fact what happens in compaction experiments. Consequently, the steady distribution is characterized by the compactivity instead of a generalized fugacity. The steady distribution function has been derived and the compactivity identified in terms of the parameters defining the mesoscopic dynamics of the model. It has been found that the results are consistent with Edwards' thermodynamical theory of powders. Nevertheless, since the model is formulated in the context of weak and short tapping, it is in fact quite doubtful that the same conclusions were reached from a generalization to stronger tapping processes. Let us point out that this would require to modify the formulation of our model by including the possibility that a lattice region would experiment several elementary excitations during the same tap.

The relationship between closed and open models, and between compactivity and fugacity, has been discussed. At the mesoscopic level of description used in this paper, the expression of one of them in terms of the transition rates cannot be inferred from the expression of the other. Nevertheless, it is true that they correspond to different derivatives of the same entropy function, like in usual thermal systems.

The model presented here can be easily generalized to mixtures of several kinds of grains, then allowing the study of segregation phenomena. Also, it can be useful to investigate the validity of the Edwards theory in this case, and eventually its possible generalizations, for instance, by extending the number of parameters needed to characterize the steady state of the mixture, as has been recently proposed $[16,15]$.

\section{ACKNOWLEDGMENTS}

We acknowledge support from the Ministerio de Ciencia y Tecnología (Spain) through Grant No. BFM2002-00303 (partially financed by FEDER funds).
[1] J.B. Knight, C.G. Fandrich, C.N. Lau, H.M. Jaeger, and S.R. Nagel, Phys. Rev. E 51, 3957 (1995).

[2] E.R. Nowak, J.B. Knight, M. Povinelli, H.M. Jaeger, and S.R. Nagel, Powder Technol. 94, 79 (1997).

[3] E.R. Nowak, J.B. Knight, E. Ben-Naim, H.M. Jaeger, and S.R. Nagel, Phys. Rev. E 57, 1971 (1998).

[4] S.F. Edwards and R.B.S. Oakeshott, Physica A 157, 1080 (1989); S.F. Edwards and A. Metha, Journal de Physique 50, 2489 (1989); S.F. Edwards and C.C. Mounfield, Physica A 210, 279 (1994).

[5] A. Coniglio and M. Nicodemi, Physica A 296, 451 (2001).

[6] H.A. Makse and J. Kurchan, Nature (London) 415, 614 (2002).
[7] A. Barrat, J. Kurchan, V. Loreto, and M. Sellitto, Phys. Rev. Lett. 85, 5034 (2000); Phys. Rev. E 63, 051301 (2001); V. Colizza, A. Barrat, and V. Loreto, Phys. Rev. E 65, 050301 (2002).

[8] A. Fierro, M. Nicodemi, and A. Coniglio, Europhys. Lett. 59, 642 (2002).

[9] D.S. Dean and A. Lefevre, Phys. Rev. Lett. 90, 198301 (2003).

[10] J.J. Brey, A. Prados, and B. Sánchez-Rey, Phys. Rev. E 60, 5685 (1999); Physica A 275, 310 (2000).

[11] A. Léfevre and D.S. Dean, J. Phys. A 34, L213 (2001).

[12] J. Berg, S. Franz, and M. Sellito, Eur. Phys. J. B 26, 349 (2002). 
[13] G. De Smedt, C. Godreche, and J.M. Luck, Eur. Phys. J. B 27, 363 (2002).

[14] A. Prados and J.J. Brey, Phys. Rev. E 66, 041308 (2002).

[15] A. Prados and J.J. Brey, Europhys. Lett. 64, 29 (2003).

[16] M. Nicodemi, A. Fierro, and A. Coniglio, Europhys. Lett. 60, 684 (2002).
[17] N.G. van Kampen, Stochastic Processes in Physics and Chemistry (North-Holland, Amsterdam, 1992).

[18] D.A. Head, Phys. Rev. E 62, 2439 (2000).

[19] A. Lefèvre, J. Phys. A 35, 9037 (2002).

[20] R. Monasson and O. Pouliquen, Physica A 236, 395 (1997). 